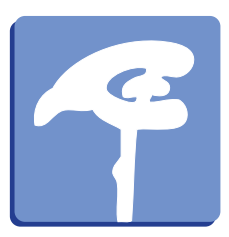

CEREBROSPINAL FLUID RESEARCH

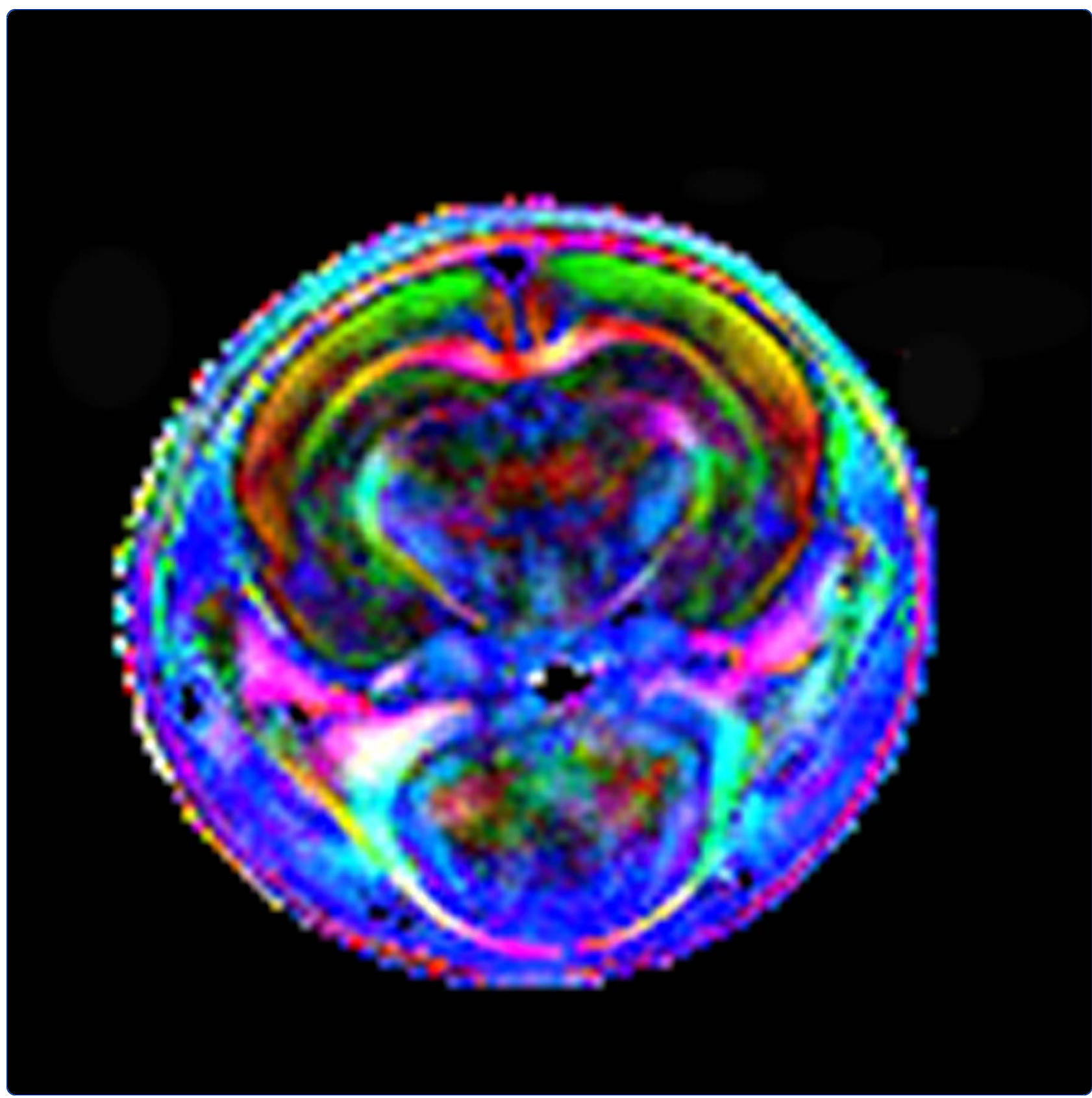

Diffusion tensor imaging correlates with cytopathology in a rat model of neonatal hydrocephalus

Yuan et al. 


\title{
Diffusion tensor imaging correlates with cytopathology in a rat model of neonatal hydrocephalus
}

\author{
Weihong Yuan ${ }^{1}$, Kelley E Deren ${ }^{2}$, James P McAllister $\|^{2}$, Scott K Holland ${ }^{1}$, Diana M Lindquist ${ }^{3}$, \\ Alessandro Cancelliere ${ }^{4}$, Melissa Mason ${ }^{2}$, Ahmed Shereen ${ }^{3}$, Dean A Hertzler ${ }^{4}$, Mekibib Altaye ${ }^{5}$, \\ Francesco $T$ Mangano ${ }^{4^{*}}$
}

\begin{abstract}
Background: Diffusion tensor imaging (DTI) is a non-invasive MRI technique that has been used to quantify CNS abnormalities in various pathologic conditions. This study was designed to quantify the anisotropic diffusion properties in the brain of neonatal rats with hydrocephalus (HCP) and to investigate association between DTI measurements and cytopathology.

Methods: DTI data were acquired between postnatal day 7 (P7) and P12 in 12 rats with HCP induced at P2 and in 15 age-matched controls. Animals were euthanized at P11 or P22/P23 and brains were processed with immunohistochemistry for glial fibrillary acidic protein (GFAP), ionized calcium-binding adaptor molecule (Iba-1), and luxol fast blue (LFB) to assess astrocytosis, microglial reactivity and degree of myelination, respectively.

Results: Hydrocephalic rats were consistently found to have an abnormally low (at corrected $p$-level of <0.05) fractional anisotropy (FA) value and an abnormally high mean diffusivity (MD) value in the cerebral cortex (CX), the corpus callosum (CC), and the internal capsule (IC). Immunohistochemical analysis demonstrated trends of increasing astrocyte and microglial reactivity in HCP rats at P11 that reached statistical significance at P22/P23. A trend toward reduced myelination in the HCP rats was also found at P22/P23. Correlation analysis at P11 for the CC demonstrated statistically significant correlations (or trends) between the DTI measurement (the decreased FA and increased MD values) and the GFAP or Iba-1 rankings. The immunohistochemical rankings in the IC at P22/P23 were also significantly correlated or demonstrated a trend with both FA and MD values.

Conclusions: This study demonstrates the feasibility of employing DTI on the brain in experimental hydrocephalus in neonatal rats and reveals impairments in multiple regions of interest in both grey and white matter. A strong correlation was found between the immunohistochemical results and the changes in anisotropic diffusion properties.
\end{abstract}

\section{Background}

Hydrocephalus (HCP) is the most common condition treated in pediatric neurosurgery [1]. The ventricular system enlarges in response to abnormal cerebrospinal fluid (CSF) dynamics. As a consequence of this imbalance, intra-cranial pressure becomes elevated, ventricles enlarge, and brain anatomy becomes distorted and

\footnotetext{
* Correspondence: francesco.mangano@cchmc.org

${ }^{4}$ Division of Pediatric Neurosurgery, University of Cincinnati, Cincinnati Children's Hospital Medical Center MLC 2016, 3333 Burnet Avenue,

Cincinnati, OH 45229, USA

Full list of author information is available at the end of the article
}

compressed. If left untreated HCP may be fatal [1]. However, long before this end-point is reached, injury to grey matter (GM) and white matter (WM) structures can cause significant neurologic deficits. Behavioral and cognitive delays typically accompany the brain abnormalities associated with HCP. The current standard of care is based on CSF diversion to extra-neural compartments via shunt surgery or via third ventriculostomy. Even though this approach has greatly reduced morbidity and mortality, much of the pathophysiology associated with this condition is yet to be fully understood.

\section{Biomed Central}

(c) 2010 Yuan et al; licensee BioMed Central Ltd. This is an Open Access article distributed under the terms of the Creative Commons Attribution License (http://creativecommons.org/licenses/by/2.0), which permits unrestricted use, distribution, and reproduction in any medium, provided the original work is properly cited. 
Diffusion tensor imaging (DTI) is an advanced magnetic resonance imaging technique that measures in vivo tissue anisotropic diffusion properties [2-9]. DTI provides information not only about the magnitude, but also the directionality of water molecule displacement in tissue [5,10-12]. The effect of water molecules interacting with specific tissue structures is reflected in a diffusion image that can reveal the characteristics of the architectural organization in a physiological or pathological environment. In clinical research, DTI has been proven to be a sensitive and specific non-invasive imaging tool for determining brain damage and recovery in various neurological and pathological disorders [13-17]. Recently, DTI has also been applied in clinical studies of $\mathrm{HCP}$ to investigate the underlying mechanisms of injury associated with poor functional outcome [18-21]. However, the validation of DTI and the association of this non-invasive, macroscopic imaging biomarker with cellular level mechanisms for WM degeneration in HCP must be supported with cytopathology. This has yet to be established. To validate DTI as a biomarker for use in childhood HCP we have used a rat model [22] in which obstructive hydrocephalus can be induced reliably and cytopathology and neurobehavioral outcomes in normal, untreated, and treated hydrocephalic animals can be monitored closely and correlated at specific time points. The animal studies can be translated to the clinical setting by comparing common characteristic changes in DTI abnormalities in the context of the developing CNS. Ultimately, a better understanding of the tissue characteristics and mechanisms underlying DTI data will lead to more effective use of DTI in treatment decisions for the pediatric patient population.

The present study was designed to quantify the anisotropic diffusion properties in neonatal HCP rat brain using DTI and to investigate their association with underlying cytopathological changes. We hypothesized that the HCP rats will demonstrate abnormal anisotropic diffusion properties as measured by decreased fractional anisotropy (FA) and increased mean diffusivity (MD) when compared to the control group. We further hypothesized that these changes will correlate with underlying microstructural alterations seen in analysis of fixed tissue. The results of these studies will further our understanding of the use of DTI in the developing CNS and associated changes in $\mathrm{HCP}$

\section{Methods}

Animals

Two groups of neonatal Sprague-Dawley rat pups were used in the study (Table 1). The first group included 12 rats with acquired hydrocephalus. The second group of controls consisted of 15 rats including 12 rats that received a sham injection of saline into the cisterna magna, plus 2 that were cooled in an ice-bath without injection, and 1 that was intact. None of the control rats developed hydrocephalus.

All animal procedures were performed according to the guidelines of the Institutional Animal Care and Use Committees at the Cincinnati Children's Hospital Research Foundation's and the University of Utah. The procedure to induce obstructive $\mathrm{HCP}$ has been described in detail in previous publications [22]. In brief, at P2, the animals were anesthetized by hypothermia and underwent injection of kaolin into the cisterna magna. MRI/DTI scans were performed between $\mathrm{P} 7$ and $\mathrm{P} 12$ at Cincinnati Children's Hospital Medical Center (CCHMC). At P11 (4 HCP rats, 4 control rats) or P22/23 (4 HCP rats, 9 control rats), the animals were euthanized and fixed by intracardiac perfusion. For this procedure, animals were first perfused with $0.9 \%$ normal saline followed by $4 \%(\mathrm{w} / \mathrm{v})$ paraformaldehyde (PFA) in phosphate buffered saline through a left intraventricular injection. The brains were subsequently post-fixed in PFA and sent to the University of Utah for immunohistochemical evaluation and cytological analysis. Six rats (4/12 HCP rats, $2 / 15$ controls) did not recover from anesthesia after the MRI/DTI scan. Although the respiration rate remained normal during the scan, these rats died under the anesthetic shortly after the scan and were not subjected to immunohistochemistry procedures (Table 1).

\section{Magnetic resonance and diffusion tensor imaging}

Prior to scanning, the rats were anesthetized with $4 \%$ Isoflurane, placed in the supine position in the magnet, and maintained sedated with a mixture of $1-1.5 \%$ isoflurane and $\mathrm{O}_{2}$ for the duration of the experiment. They were kept warm, at approximately $37^{\circ} \mathrm{C}$, with circulating warmed air. A bite bar was used to stabilize the head. The respiratory rate was monitored closely and maintained at 50 breaths/min $( \pm 10$ breaths $/ \mathrm{min})$.

All MRI/DTI imaging data were acquired on a 7T Bruker MRI scanner (Bruker Biospec 70/30, Karlsruhe, Germany) equipped with an actively shielded $400 \mathrm{mT} / \mathrm{m}$ gradient set. The diameter of the gradient set was $20 \mathrm{~cm}$. A customdesigned single-turn-solenoid (STS) RF coil was used in this study. The detailed feature of the coil has been previously reported [23]. The coil measured $25 \mathrm{~mm}$ in diameter and $30 \mathrm{~mm}$ in length. The B1 field distribution test showed that the inhomogeneity was modest with percentage deviation of the field strength in the peripheral region at $<3 \%$ from the central value (Fig two in [23]).

DTI images were acquired in the coronal plane with a 6-direction diffusion weighted multi-slice spin-echo imaging protocol: $\mathrm{TR}=2500 \mathrm{~ms}, \mathrm{TE}=21 \mathrm{~ms}, 5$ slices, slice thickness $=1.5 \mathrm{~mm}$, inter-slice gap $=0.25 \mathrm{~mm}$, b-value $=$ $1200 \mathrm{~s} / \mathrm{mm}^{2}$, diffusion gradient duration $\delta=4 \mathrm{~ms}$, gradient separation $\Delta=12 \mathrm{~ms}$. Twenty-five of the 27 rats 
Table 1 Experimental procedures performed on each animal used in the study

\begin{tabular}{|c|c|c|c|c|c|c|c|c|c|c|c|c|}
\hline \multirow[b]{2}{*}{ Animal } & \multicolumn{12}{|c|}{ Days of Age } \\
\hline & P7 & P8 & P9 & P10 & P11 & P12 & P13 & P15 & P17 & P19 & P22 & P23 \\
\hline \multicolumn{13}{|l|}{$\mathrm{HCP}$} \\
\hline HCP_01 & & DTI & & & & & & & & $\mathrm{D}+$ & & \\
\hline HCP_02 & & & DTI & & & & & & & & & $\| H C^{*}$ \\
\hline HCP_03 & & DTI & & & & & & & Dt & & & \\
\hline HCP_04 & & & DTI & & & & & & & & & $\mathrm{H} \mathrm{C}^{*}$ \\
\hline HCP_05 & & & & & DTI & & & & & & & $\mathrm{HC}$ \\
\hline HCP_06 & & & & DTI & & & & $\mathrm{D}+$ & & & & \\
\hline HCP_07 & & & & & DTI/D & & & & & & & \\
\hline HCP_08 & DTI & & & & & & & & & & $\mathrm{HHC}^{*}$ & \\
\hline HCP_09 & & & DTI & & $\| H C^{*}$ & & & & & & & \\
\hline HCP_10 & & & DTI & & $I H C^{*}$ & & & & & & & \\
\hline HCP_11 & & & DTI & & $\mathrm{HCC}^{*}$ & & & & & & & \\
\hline HCP_12 & & & & DTI & $\mathrm{IHC}$ & & & & & & & \\
\hline \multicolumn{13}{|l|}{ Control } \\
\hline Control_01 & & DTI & & & & & & & & & & $H C^{*}$ \\
\hline Control_02 & & & DTI & & & & & & & & & $\mathrm{HC}$ \\
\hline Control_03 & & DTI & & & & & & & & & & $\mathrm{HC}$ \\
\hline Control_04 & & DTI & & & & & & & $\mathrm{D}+$ & & & \\
\hline Control_05 & DTI & & & & & & & & & & $\mathrm{IHC}$ & \\
\hline Control_06 & & & DTI & & & & & & & & $\mathrm{IHC}$ & \\
\hline Control_07 & & & DTI & & & & & & & & $\mathrm{HHC}^{*}$ & \\
\hline Control_08 & & & & DTI & & & & & & & $\mathrm{IHC}$ & \\
\hline Control_09 & & & & & & DTI/D & & & & & & \\
\hline Control_10 & & & DTI & & & & & & & & $\mathrm{IHC}^{*}$ & \\
\hline Control_11 & & & & & DTI & & & & & & & $\mathrm{HC}$ \\
\hline Control_12 & & & DTI & & $\mathrm{IHC}$ & & & & & & & \\
\hline Control_13 & & & & DTI & ${ }^{\prime H C} C^{*}$ & & & & & & & \\
\hline Control_14 & & & & DTI & $\mathrm{IHC}$ & & & & & & & \\
\hline Control_15 & & & DTI & & $\| H C^{*}$ & & & & & & & \\
\hline
\end{tabular}

Note. DTI: diffusion tensor imaging; IHC: Sacrificed by cardiac perfusion and processed for immunohistochemistry; * underwent IHC; D: Died shortly after DTI scan; Dt: Died shortly after scan, note the imaging data were not used due to severe HCP.

included in the present study were scanned at an in-plane resolution of $200 \times 200 \mu \mathrm{m}$ with four different fields of view (FOV) between $16 \times 16 \mathrm{~mm}$ and $25.6 \times 25.6 \mathrm{~mm}$. No statistical significance was found between the hydrocephalic group and the control group in terms of the frequency of occurrence of these four different FOVs based on the Freeman-Halton extension of the Fisher exact probability test [24]. The time for acquisition ranged from 23:20 min to $37: 20 \mathrm{~min}$. The remaining two rats were scanned at slightly different in-plane resolutions (resolution $=196 \times$ $196 \mu \mathrm{m}, \mathrm{TA}=26: 50 \mathrm{~min} ., \mathrm{n}=1$; resolution $=200 \times 225$ $\mu \mathrm{m}, \mathrm{TA}=23: 20 \mathrm{~min} ., \mathrm{n}=1$ ) and no significant difference was identified with DTI measurement.

T2-weighted images of the whole brain were acquired using rapid acquisition with relaxation enhancement
(RARE) sequence with the following parameters: $\mathrm{TR}=$ $1000 \mathrm{~ms}$; effective TE $=70.56 \mathrm{~ms}$; FOV $=32 \times 19.2 \times$ $19.2 \mathrm{~mm}$; acquisition matrix $=256 \times 128 \times 128$; spatial resolution $=0.125 \times 0.150 \times 0.150 \mathrm{~mm}$; scanning time $=$ 17:04 min. The T2-weighted RARE anatomical images were used to assess the ventricular size and also as a reference for anatomical structures.

\section{Immunohistochemistry}

Brain tissue was processed from 2 control and $3 \mathrm{HCP}$ rats sacrificed at $\mathrm{P} 11$ and 3 control and $3 \mathrm{HCP}$ rats sacrificed at P22 or P23 (Table 1). Ten additional rats were perfused and fixed but did not undergo immunohistochemical analysis. Two control and 2 HCP rats sacrificed at P21 in previous experiments (not included 
in Table 1) were included for comparison in the present study. These additional animals underwent the same surgical procedures that have been described with the exception of the timing of kaolin injection or sham injection: the injection occurred at P1. They were not subjected to MRI/DTI scan thus were not included in the correlation analysis. Post-fixed brains were cut coronally into 3 blocks containing the frontal, parietal and occipital cortices. These blocks were embedded in paraffin, and sectioned serially at a thickness of $15 \mu \mathrm{m}$. Every $10^{\text {th }}$ section was selected in order to collect 12 sets of adjacent sections. Using established protocols, the following immunohistochemical stains were applied: (1) luxol fast blue (LFB), a sensitive staining method that detects mature myelin [25,26]; (2) glial fibrillary acidic protein (GFAP), a marker for both resting and reactive astrocytes [27]; and (3) ionized calcium-binding adaptor molecule (Iba-1), a marker for both resting and reactive microglia [28].

LFB staining was performed by de-paraffinizing and rehydrating the tissue. Slides were then incubated in the LFB stain for $2 \mathrm{~h}$ at $60^{\circ} \mathrm{C}$. The sections were then rinsed in $95 \% \mathrm{ETOH}, \mathrm{dH}_{2} \mathrm{O}$ and differentiated with $0.05 \%$ lithium carbonate. Once the appropriate intensity of LFB stain was achieved, the sections were rinsed in distilled $\mathrm{H}_{2} \mathrm{O}, 70 \% \mathrm{ETOH}$, and then counterstained with cresyl violet.

Tissue sections were processed for GFAP immunohistochemistry according to previously described methods [29]. In summary, sections were rehydrated and antigen retrieval was performed. Prior to incubation (90 min) with the primary GFAP antibody (1:1000 Dako Cytomation, Glostrup, Denmark), sections were blocked with $\mathrm{H}_{2} \mathrm{O}_{2}$. The tissue was washed with phosphate buffered saline with $0.1 \%$ triton $C$ (PBS-t) and the secondary antibody (1:200, Vector Laboratories, Burlingame, CA, USA) was applied for $45 \mathrm{~min}$ at room temperature. Following the secondary antibody Avidin Biotinylated enzyme Complex (ABC) was added for $30 \mathrm{~min}$, washed with PBS-t and developed with diaminobenzidine (DAB). Sections were counterstained with cresyl violet.

Immunostaining for Iba-1 followed the same procedures described for GFAP with the exception that antiIba-1 (1: 500 WAKO Chemicals, Richmond, USA) was the primary antibody.

Stained sections were analyzed using a semi-quantitative scale described in detail in a recent report from our group [30]. Briefly, the scales pertaining to the GFAP and Iba-1 stains were based on cellular morphology and density. A score of 0 represented a resting state and a score of 3 represented a severely reactive state. The scale pertaining to the LFB stains was based on the intensity of the stained myelin. A score of 0 on the LFB scale represented a complete lack of myelin and a score of 3 indicated full myelination.

\section{DTI data processing and analysis}

DTI data processing and analysis methodology details have been described in our previous publications [31,32]. The procedure is briefly summarized as follows. Image reconstructions, post-processing, and ROI-based DTI parameter calculations were performed with the software DTIStudio 2.4 [33]. On a pixel by pixel basis, the six elements (Dxx, Dyy, Dzz, Dxy, Dxz, and Dyz) were calculated and diagonalized to compute the three eigenvalues $\left(\lambda_{1}, \lambda_{2}, \lambda_{3}\right)$ corresponding to the three eigenvectors in the diffusion tensor matrix. Mean diffusivity (MD) was calculated as the mean of the three eigenvalues $\left(\mathrm{MD}=\left(\lambda_{1}+\right.\right.$ $\left.\lambda_{2}+\lambda_{3}\right) / 3$ ). Fractional anisotropy (FA) was calculated on a pixel-wise basis, using standard methods [4,12].

As shown in Figure 1, a color-coded FA map was used to identify regions of interest (ROI). DTI parameters were calculated for respective ROIs individually in each section sampled. The ROIs for each subject were manually determined by an operator (WY) on color-coded FA and MD maps and anatomical images under the guidance of a neuroanatomist (JPM) and a pediatric neurosurgeon (FTM), (Figures 1 and 2). For each rat, the following ROIs were defined: corpus callosum (CC), internal capsule (IC), external capsule (EC), cerebral cortex $(\mathrm{CX})$, caudate-putamen complex $(\mathrm{CPu})$, hippocampus (HC), and fornix (FX). The ROI in the EC in hydrocephalic rats was found to be very difficult to delineate reliably, thus it was not included in the data analysis comparing HCP rats and control rats. EC of normal controls was still included in the data analysis when the age correlation of DTI parameters in various ROIs was studied in order to assess the developmental impact.

\section{Statistical analyses}

Before conducting any statistical analysis, the distribution of the outcome data was examined for normality and the outcome variables that exhibit deviation from normality were transformed using a Box-Cox transformation [34]. For some of the outcome variables a suitable transformation was not found to satisfy the normality assumption. For uniformity we ran all statistical analysis using nonparametric methods. Wilcoxon two-sample test was used to compare the DTI parameters between HCP rats and the age matched controls over the period between P7 and P12. Although age is matched between the two groups, its potential impact on the outcome variables was examined using a Spearman rank correlation followed by regression analysis that contained both the comparison groups and age in the same model. We also ran a sub-group analysis 


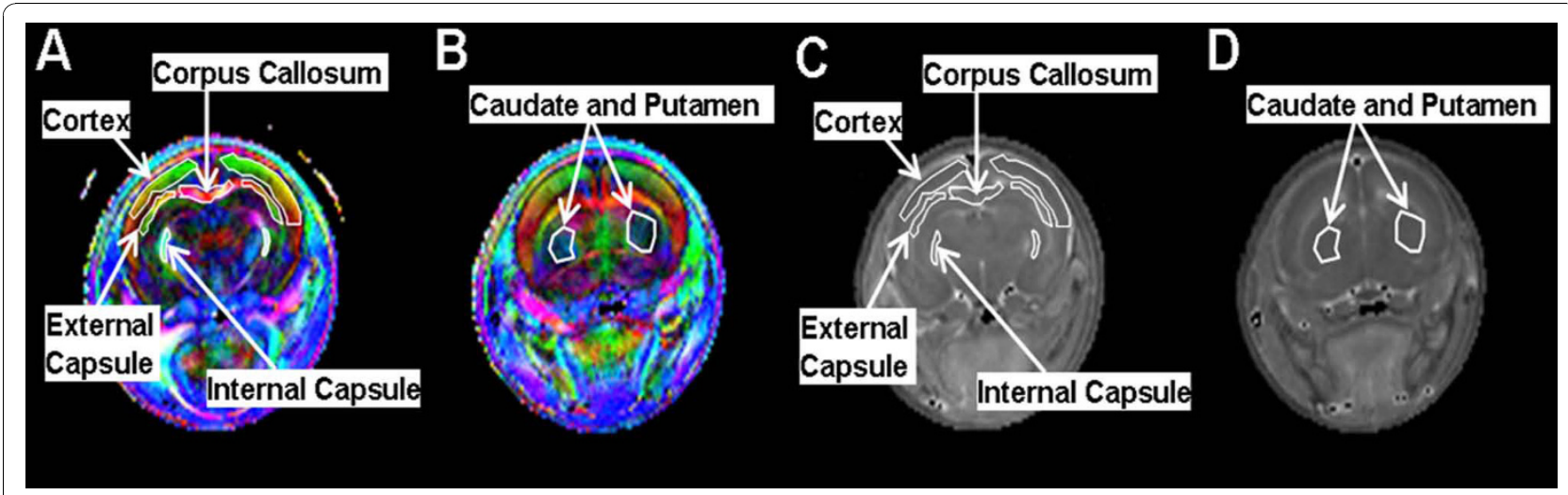

Figure 1 In vivo diffusion tensor imaging of a control rat at postnatal day $\mathbf{8}$. In the fractional anisotropy-based color coded axis maps (A and B), the colors are used to indicate the preferred diffusion direction in a coronal plane. Red, green, and blue represent transverse, dorsoventral and rostro-caudal directions, respectively. The brightness is coded by the FA value. On this map, both white matter (corpus callosum, internal capsule, and external capsule) and grey matter (cortex, caudate and putamen) can be identified. C and D are mean diffusivity maps showing the same structures.

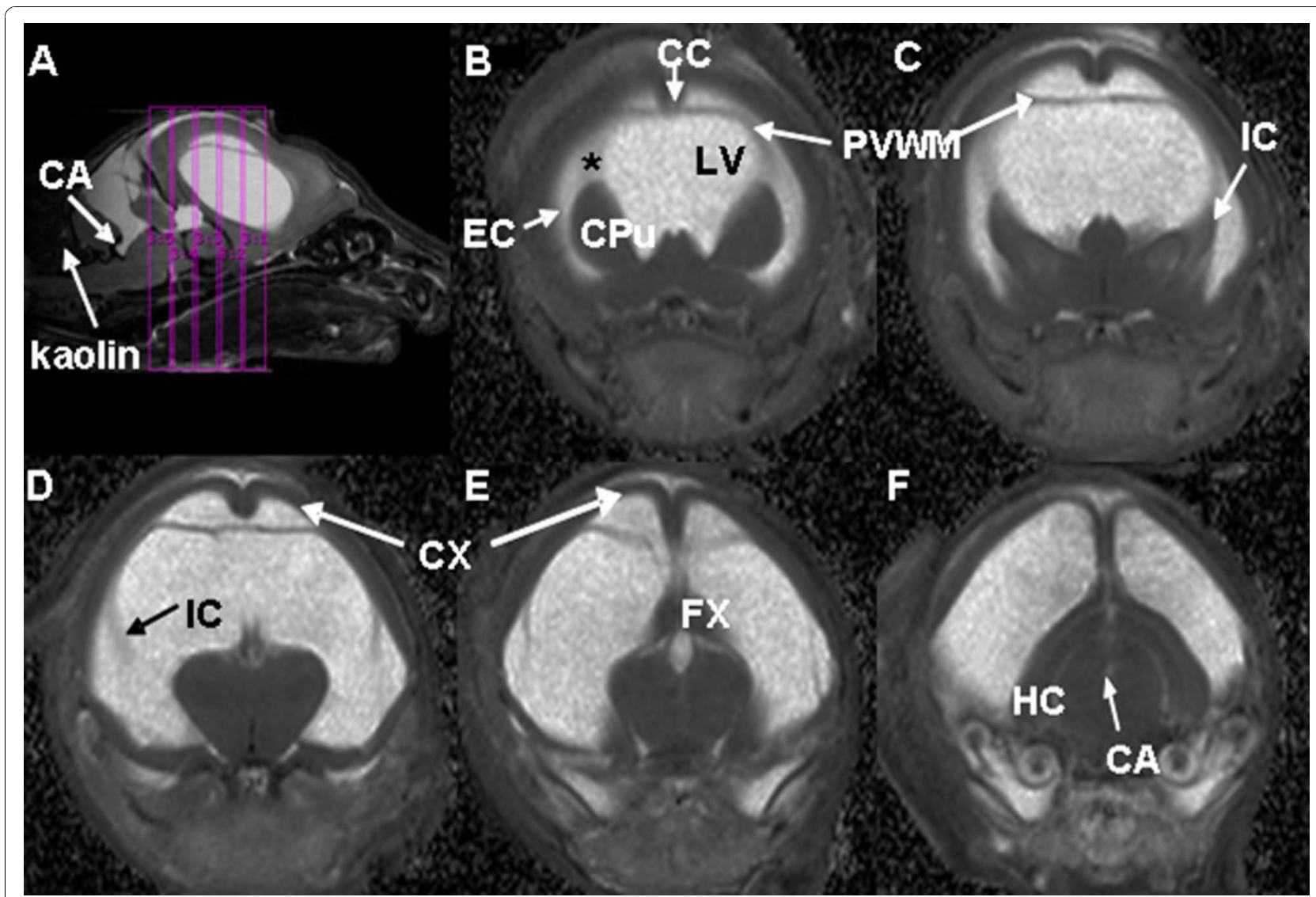

Figure 2 Mean diffusivity maps of hydrocephalic rats at postnatal day 11. The mid-sagittal image (A) shows the location of the five coronal slices (B-F) arranged from rostral to caudal. The expansion of the lateral ventricle and the posterior recess of the cerebral aqueduct (CA) can also be seen. Abbreviations: CC, corpus callosum; CPu, caudate-putamen; CX, cortex; EC, external capsule; FX, fornix; IC, internal capsule; HC, hippocampus; LV, lateral ventricle; PVWM, periventricular white matter. 
Table 2 Comparison of DTI parameters (fractional anisotropy, FA and mean diffusivity, MD) between hydrocephalic (HCP) and control groups between postnatal days 7 and 12

\begin{tabular}{|c|c|c|c|}
\hline & $\mathrm{HCP}(n=12)$ & Control $(n=15)$ & Corrected p-value \\
\hline \multicolumn{4}{|l|}{$\overline{c x}$} \\
\hline FA & $0.17 \pm 0.03$ & $0.25 \pm 0.04$ & $<0.01^{*}$ \\
\hline MD & $1.22 \pm 0.09$ & $1.12 \pm 0.10$ & $<0.05^{*}$ \\
\hline
\end{tabular}

$\mathrm{CPu}$

$\begin{array}{llll}\text { FA } & 0.22 \pm 0.05 & 0.20 \pm 0.05 & \text { NS } \\ \text { MD } & 1.23 \pm 0.10 & 1.17 \pm 0.13 & \text { NS }\end{array}$

CC

$\begin{array}{llll}\text { FA } & 0.29 \pm 0.05 & 0.47 \pm 0.08 & <0.01^{*} \\ \text { MD } & 2.25 \pm 0.32 & 1.25 \pm 0.15 & <0.01^{*}\end{array}$

IC

$\begin{array}{llll}\text { FA } & 0.23 \pm 0.04 & 0.31 \pm 0.04 & <0.01 * \\ \text { MD } & 2.39 \pm 0.22 & 1.17 \pm 0.09 & <0.01 *\end{array}$

HC

$\begin{array}{clll}\text { FA } & 0.19 \pm 0.03 & 0.17 \pm 0.04 & \text { NS } \\ \text { MD } & 1.19 \pm 0.10 & 1.10 \pm 0.26 & \text { NS }\end{array}$

FX

$\begin{array}{llll}\text { FA } & 0.20 \pm 0.04 & 0.24 \pm 0.05 & \text { NS } \\ \text { MD } & 1.27 \pm 0.10 & 1.18 \pm 0.11 & \text { NS }\end{array}$

Note: * significantly different (Wilcoxon two-sample test). Numbers are mean \pm standard deviation. MD is in the unit of $10^{-3} \mathrm{~mm}^{2} / \mathrm{s}$. NS: not significant, brain regions as for Figure 2.

using Wilcoxon two-sample test to compare the DTI parameters between HCP rats and controls at P9. Immunohistochemical results were also analyzed using Wilcoxon two-sample test. In addition, the Spearman correlation
Table 3 Comparison of DTI parameters (fractional anisotropy, FA and mean diffusivity, MD) between HCP and control groups at postnatal day 9

\begin{tabular}{ccccc}
\hline & & HCP $(\mathbf{n}=\mathbf{5})$ & Control $(\mathbf{n}=\mathbf{6})$ & Corrected $\mathbf{p}$-value \\
\hline CX & & & & \\
& FA & $0.16 \pm 0.01$ & $0.25 \pm 0.05$ & $<0.05^{*}$ \\
& MD & $1.22 \pm 0.07$ & $1.17 \pm 0.14$ & NS
\end{tabular}

$\mathrm{CPu}$

$\begin{array}{llll}\text { FA } & 0.22 \pm 0.07 & 0.21 \pm 0.06 & \text { NS } \\ \text { MD } & 1.26 \pm 0.10 & 1.21 \pm 0.17 & \text { NS }\end{array}$

CC

$\begin{array}{llll}\text { FA } & 0.28 \pm 0.03 & 0.44 \pm 0.07 & <0.05^{*} \\ \text { MD } & 2.44 \pm 0.18 & 1.27 \pm 0.13 & <0.05^{*}\end{array}$

IC

$\begin{array}{cccc}\text { FA } & 0.24 \pm 0.03 & 0.28 \pm 0.05 & \text { NS } \\ \text { MD } & 2.37 \pm 0.17 & 1.21 \pm 0.15 & <0.05^{*}\end{array}$

HC

$\begin{array}{cccc}\text { FA } & 0.2 \pm 0.04 & 0.17 \pm 0.04 & \text { NS } \\ \text { MD } & 1.25 \pm 0.04 & 0.94 \pm 0.45 & \text { NS }\end{array}$

FX

$\begin{array}{cccc}\text { FA } & 0.20 \pm 0.05 & 0.28 \pm 0.03 & <0.05^{*} \\ \text { MD } & 1.26 \pm 0.05 & 1.21 \pm 0.10 & \text { NS }\end{array}$

Note: * significantly different (Wilcoxon two sample test). Numbers are mean \pm standard deviation. $\mathrm{MD}$, is in the unit of $10^{-3} \mathrm{~mm}^{2} / \mathrm{s}$. NS: not significant, brain regions as for figure 2 .

was used in the correlation analysis between DTI parameters and the immunochemistry ranking scales. To account for the expected proportion of incorrectly rejected null hypotheses (Type I error rate) due to multiple testing, we made a correction for multiple comparisons using the
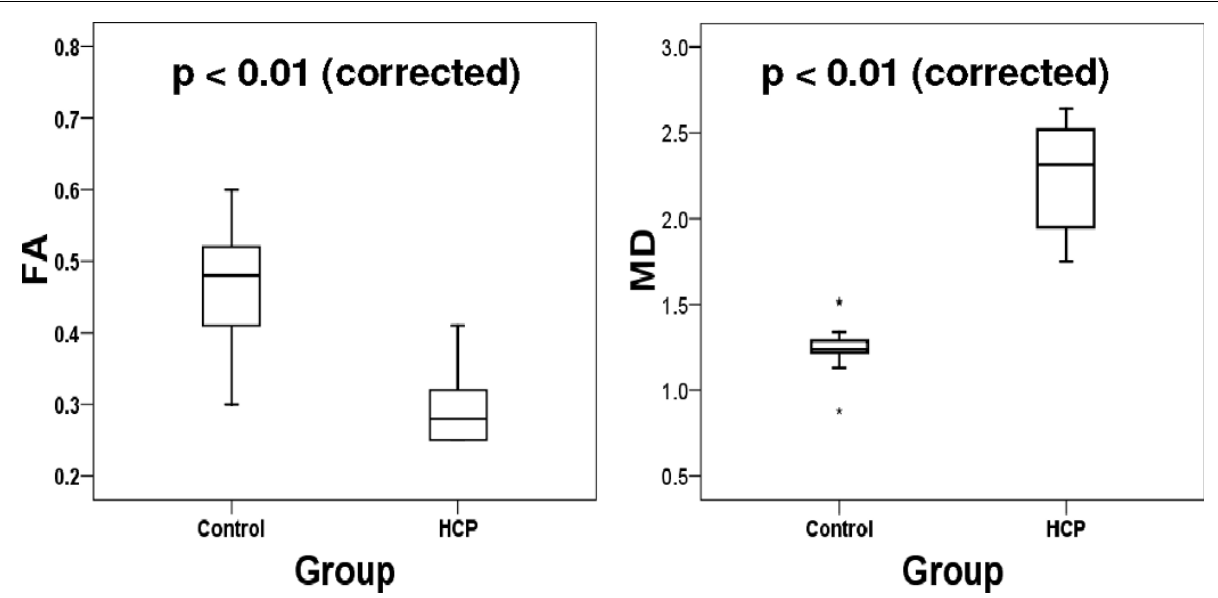

Figure 3 Box-plots comparing the fractional anisotropy (FA) and mean diffusivity (MD, $10^{-3} \mathrm{~mm}^{2} / \mathrm{s}$ ) values in corpus callosum in hydrocephalic rats and the control rats. All ages combined, $n=12$ for hydrocephalic and $n=15$ for control rats. The Wilcoxon two-sample test showed a significant difference ( $p<0.05$, corrected for multiple comparison). 
False Discovery Rate (FDR) method [35] in all the statistical tests except for the correlation analysis between DTI and cytopathology. All statistical analyses were done using SAS v.9.2 (SAS Institute Inc., Carey, NC, USA) or SPSS v.15 (Chicago, IL, USA).

\section{Results}

Abnormal DTI measurements in neonatal rats with hydrocephalus

DTI data acquired between P7 and P12 were compared between the control $(n=15)$ and the HCP group $(n=12)$. In the regression analysis, where comparison group and age were considered in the same model, age did not contribute significantly to the change in DTI measurements. Therefore, the two groups were compared with Wilcoxon independent two-sample test. Significant differences in DTI parameters were found in both GM and WM (Table 2, Figure 3). Among the GM ROIs, the FA of the HCP group in CX was significantly lower $(p<0.01)$ and the MD significantly higher $(p<0.05)$ than those in the control group. In the WM ROIs, statistically significant decreases in FA values $(p<0.01$ for both CC and IC) and increases in MD values $(p<0.01$ for both CC and IC) were found in the HCP group.

To explore the earliest time at which the impact of $\mathrm{HCP}$ can be reflected in DTI parameters, we compared
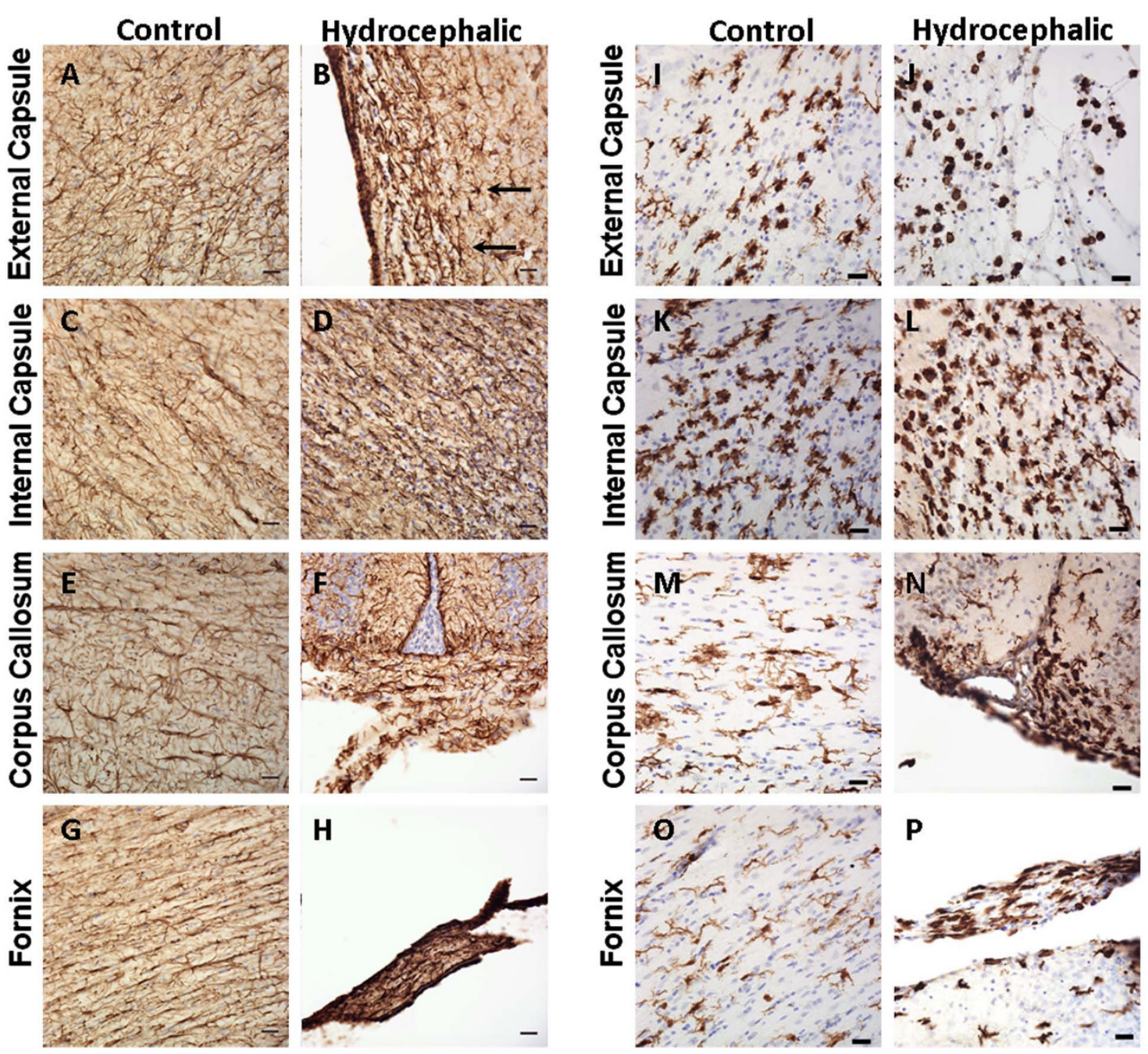

Figure 4 Representative photomicrographs of GFAP for astrocytes (A-H) and Iba-1 for microglia (I-P) immunohistochemistry from the external capsule, internal capsule, corpus callosum, and fornix in P11 saline control animals (A, C, E, G for GFAP; I, K, M, O for Iba-1) and hydrocephalic animals (B, D, F, H for GFAP; J, L, N, P for Iba-1). For GFAP, despite the immaturity of the tissue, the hydrocephalic animals exhibit the same severe glial reaction observed in the P22/23 animals as evidenced by large intensely stained cell bodies and thick processes ( $B$, straight arrows). For Iba-1, note the round intensely stained profiles that represent highly phagocytic microglial somata in the white matter of control animals. These profiles are increased in hydrocephalic animals at this age, but are not found in any 22 or 23 -day old animals. Scale bar $=25 \mu \mathrm{m}$ and cresyl violet counterstain for all panels. 


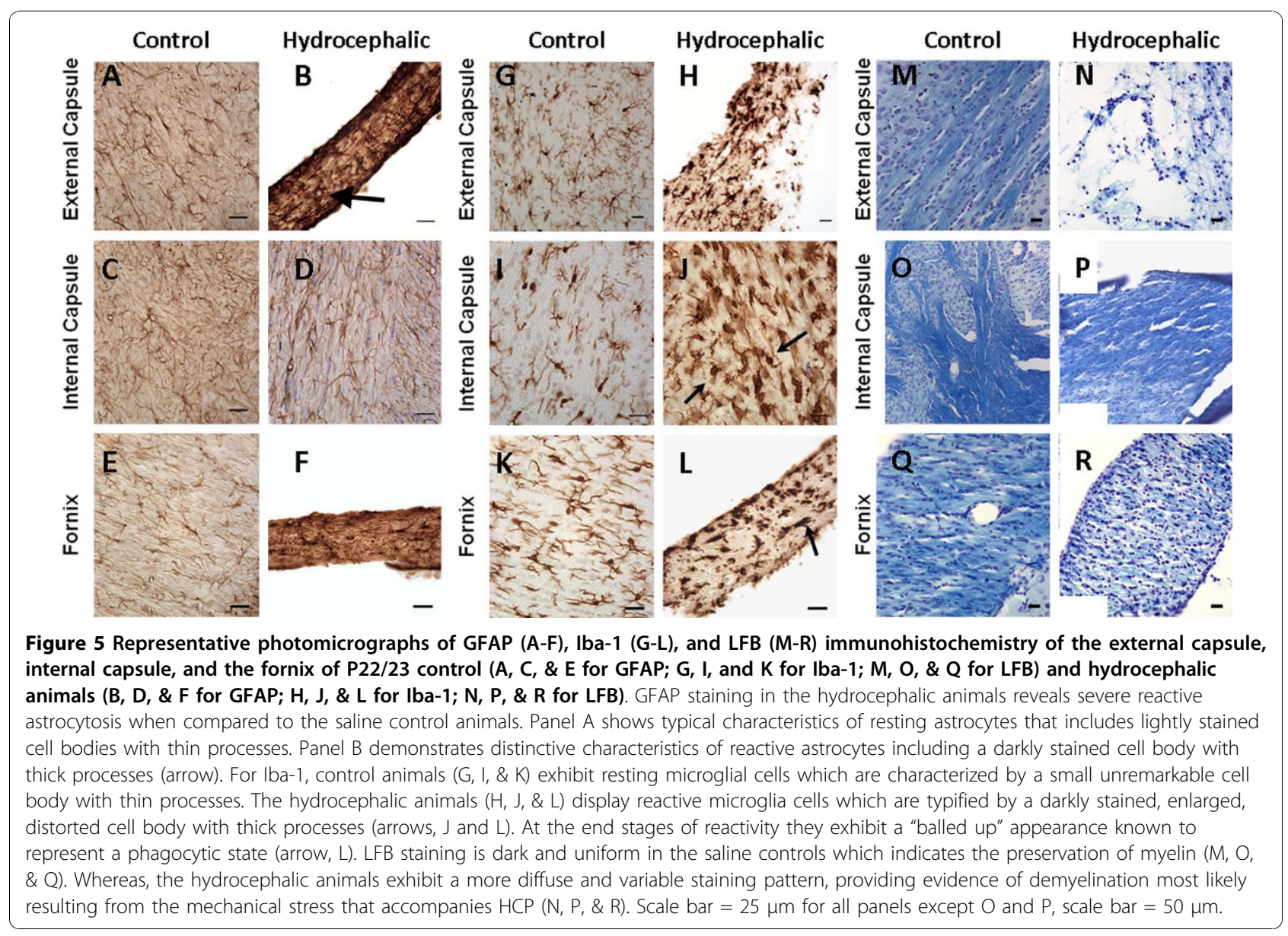

FA and MD values between the two groups for each individual postnatal day. Only the difference at P9 reached statistical significance (Table 3 ). The Wilcoxon two-sample tests were performed for all DTI values in each ROI as described in the Methods section. The FA values of the $\mathrm{CX}$ in the $\mathrm{HCP}$ group were significantly lower compared to the controls $(p<0.05)$. In the WM, specifically the CC, the FA in HCP rats was significantly lower than that in the controls $(p<0.05)$ and the MD was significantly increased $(p<0.05)$. In the IC, the MD value was abnormally high in the HCP rats $(p<0.05)$, but their FA values were within normal range. In the FX, the FA values of the HCP rats were abnormally high $(p<0.05)$ with the MD value within normal range. No statistically significant difference was identified for the other two ROIs $(\mathrm{CPu}, \mathrm{HC}$, Table 3$)$.

\section{Immunohistochemical results}

Overall, hydrocephalic animals at both P11 (Figure 4) and P22/23 (Figure 5) displayed increases in astrocyte and microglial responses and a decrease in myelination in all WM regions studied. Numerous GFAP- and Iba- 1-positive cells were found, and many of these astrocytes and microglia exhibited the large soma and thickened processes characteristic of reactive glia.

Quantitative rankings (Table 4) confirmed a trend of increased astrocyte reactivity (GFAP staining) at P11 in IC, EC, FX, and CC. At P22/P23 the same regions reached statistical significance $(p<0.05)$, with the exception of the corpus callosum which could not be analyzed as it was too thin or missing altogether.

Microglial reactivity followed the same trend of increases in the IC, EC, FX, and CC (Table 4). At P22/ P23, microglial reactivity also reached statistical significance $(p<0.05)$ with the exception of the CC which could not be analyzed.

Myelination, as visualized with LFB staining, was undetectable in both the control and hydrocephalic animals at P11. At P22/P23 myelination had matured to the point where it could be detected histologically, and although there was a consistent trend toward less staining in the IC, EC, and FX of hydrocephalic animals these changes were only statistically significant in the IC, $p<0.05$ (Figure 5 and Table 4). 
Table 4 Cytological rankings in hydrocephalic and control rats at P11 and P22/P23 as determined from immunohistochemical analysis

\begin{tabular}{|c|c|c|c|c|c|c|c|}
\hline & & \multicolumn{3}{|c|}{ P11 } & \multicolumn{3}{|c|}{ P22/P23 } \\
\hline & & $\mathrm{HCP}(n=3)$ & Control $(n=2)$ & Corrected p-value & $\mathrm{HCP}(n=5)$ & Control $(n=5)$ & Corrected $p$-value \\
\hline \multicolumn{8}{|c|}{ IC } \\
\hline & GFAP & $2.67 \pm 0.58$ & $0.5 \pm 0.00$ & NS & $2.80 \pm 0.28$ & $0.25 \pm 0.30$ & $<0.05^{*}$ \\
\hline & lba-1 & $2.67 \pm 0.29$ & $2.0 \pm 0.00$ & NS & $2.90 \pm 0.22$ & $0.90 \pm 0.58$ & $<0.05^{*}$ \\
\hline & LFB & $n / d$ & $n / d$ & $n / d$ & $1.69 \pm 0.51$ & $2.80 \pm 0.21$ & $<0.05^{*}$ \\
\hline \multicolumn{8}{|c|}{ EC } \\
\hline & GFAP & $3.00 \pm 0.00$ & $0.69 \pm 0.27$ & NS & $3.00 \pm 0.00$ & $0.18 \pm 0.14$ & $<0.05^{*}$ \\
\hline & lba-1 & $3.00 \pm 0.00$ & $1.5 \pm 0.00$ & NS & $3.00 \pm 0.00$ & $0.40 \pm 0.39$ & $<0.05^{*}$ \\
\hline & LFB & $n / d$ & $n / d$ & $n / d$ & $1.60 \pm 1.04$ & $2.15 \pm 0.73$ & NS \\
\hline \multicolumn{8}{|c|}{ FX } \\
\hline & GFAP & $3.00 \pm 0.00$ & $0.88 \pm 0.53$ & NS & $2.75 \pm 0.35$ & $0.20 \pm 0.33$ & $<0.05^{*}$ \\
\hline & lba-1 & $3.00 \pm 0.00$ & $0.5 \pm 0.00$ & NS & $2.85 \pm 0.34$ & $0.15 \pm 0.14$ & $<0.05^{*}$ \\
\hline & LFB & $n / d$ & $n / d$ & $n / d$ & $1.35 \pm 0.60$ & $2.20 \pm 0.45$ & NS \\
\hline \multicolumn{8}{|c|}{$\mathrm{CC}$} \\
\hline & GFAP & $3.00 \pm 0.00$ & $0.75 \pm 0.35$ & NS & $n / d$ & $n / d$ & $n / d$ \\
\hline & lba-1 & $3.00 \pm 0.00$ & $0.75 \pm 0.35$ & NS & $n / d$ & $n / d$ & $n / d$ \\
\hline & LFB & $n / d$ & $n / d$ & $n / d$ & $n / d$ & $n / d$ & $n / d$ \\
\hline
\end{tabular}

GFAP: glial fibrillary acidic protein for astrocytes, lba-1: ionized calcium-binding adaptor molecule for microglia, LFB: luxol fast blue for myelin. Note. * significantly different (Wilcoxon two sample test); NS: not significant; n/d: not detectable; LFB stain too weak at P11 and CC too thin or missing at P22/P23. Brain regions as for figure 2.

Correlation between immunohistochemical rankings and DTI The immunohistochemical semi-quantitative scale [30] was used to correlate cytology to imaging findings (Table 5). In the IC and FX, no significant differences were identified between the DTI measurement and any of the 3 ranked immunohistochemical parameters at P11. The FA in the $C C$ was negatively correlated with the P11 GFAP ranking $(\mathrm{R}=-0.89 ; p<0.05)$ and moderately correlated with the P11 Iba-1 ranking but without statistical significance $(R=-0.78)$. Also at $P 11$, the $\mathrm{MD}$ in the $\mathrm{CC}$ was moderately correlated with GFAP but without statistical significance $(\mathrm{R}=0.78)$ and was significantly correlated with Iba-1 ranking scale $(\mathrm{R}=0.89, p<0.05$, Table 5$)$.

The ventricular enlargement in the third week and thereafter was too severe to allow for reliable imaging,

Table 5 Correlation analysis between DTI metrics and immunohistochemical ranking scores

\begin{tabular}{|c|c|c|c|c|c|c|c|c|c|c|c|c|c|}
\hline & & \multicolumn{4}{|c|}{ IC } & \multicolumn{4}{|c|}{ Fornix } & \multicolumn{4}{|c|}{$\mathrm{CC}$} \\
\hline & & \multicolumn{2}{|c|}{ P11 } & \multicolumn{2}{|c|}{ P22/P23 } & \multicolumn{2}{|c|}{ P11 } & \multicolumn{2}{|c|}{$\mathrm{P} 22 / \mathrm{P} 23$} & \multicolumn{2}{|c|}{ P11 } & \multicolumn{2}{|c|}{$\mathrm{P} 22 / \mathrm{P} 23$} \\
\hline & & $\mathbf{R}$ & $p$ & $\mathbf{R}$ & $p$ & $\mathbf{R}$ & $p$ & $\mathbf{R}$ & $p$ & $\mathbf{R}$ & $p$ & $\mathbf{R}$ & $p$ \\
\hline \multicolumn{14}{|l|}{ GFAP } \\
\hline & FA & -0.47 & NS & -0.88 & $<0.05^{*}$ & -0.48 & NS & -0.52 & NS & -0.89 & $<0.05^{*}$ & $\mathrm{n} / \mathrm{d}$ & $\mathrm{n} / \mathrm{d}$ \\
\hline & MD & 0.63 & NS & 0.79 & NS & -0.18 & NS & 0.52 & NS & 0.78 & NS & $\mathrm{n} / \mathrm{d}$ & $\mathrm{n} / \mathrm{d}$ \\
\hline \multicolumn{14}{|l|}{ Iba-1 } \\
\hline & FA & -0.26 & NS & -0.88 & $<0.05^{*}$ & -0.45 & NS & -0.62 & NS & -0.78 & NS & $\mathrm{n} / \mathrm{d}$ & $\mathrm{n} / \mathrm{d}$ \\
\hline & MD & 0.79 & NS & 0.76 & NS & -0.24 & NS & 0.49 & NS & 0.89 & $<0.05^{*}$ & $\mathrm{n} / \mathrm{d}$ & $\mathrm{n} / \mathrm{d}$ \\
\hline \multicolumn{14}{|l|}{ LFB } \\
\hline & FA & $\mathrm{n} / \mathrm{d}$ & $\mathrm{n} / \mathrm{d}$ & 0.74 & NS & $\mathrm{n} / \mathrm{d}$ & $\mathrm{n} / \mathrm{d}$ & -0.03 & NS & $\mathrm{n} / \mathrm{d}$ & $\mathrm{n} / \mathrm{d}$ & $\mathrm{n} / \mathrm{d}$ & $\mathrm{n} / \mathrm{d}$ \\
\hline & MD & $\mathrm{n} / \mathrm{d}$ & $\mathrm{n} / \mathrm{d}$ & -0.74 & NS & $n / d$ & $n / d$ & -0.40 & NS & $n / d$ & $\mathrm{n} / \mathrm{d}$ & $\mathrm{n} / \mathrm{d}$ & $\mathrm{n} / \mathrm{d}$ \\
\hline
\end{tabular}

GFAP: glial fibrillary acidic protein for astrocytes, lba-1: ionized calcium-binding adaptor molecule for microglia, LFB: luxol fast blue for myelin. Note: * significant correlation between DTI and immunohistochemical grading (Spearman test); 3 HCP and 2 control rats were used at P11; 3 HCP and 3 control rats were used at P22/P23; n/d: not detectable. 
hence the histology results acquired at P22/P23 were correlated to the DTI metrics measured between P7$\mathrm{P} 10$. The FA values in the IC were found to be significantly correlated with GFAP and Iba- 1 ( $p<0.05$, for both) suggesting perhaps that radiographic evidence of injury in this anatomic location may occur early in the process. The correlation between FA in the IC and LFB staining of the same area was moderate but it did not reach statistical significance. MD values measured from the IC were found to be moderately correlated with the rankings of all three stains but without reaching statistical significance (Table 5).

\section{Discussion}

The goal of the present study was twofold: (1). to show proof-of-principle for adopting DTI methods to study the impact of obstructive HCP on the brain in a rodent model; and (2) to evaluate the tissue integrity in the hydrocephalic brain using DTI and correlate it with cytopathology. Our results demonstrate that DTI can successfully be used to quantify the underlying structural abnormality as reflected in the changes of the regional anisotropic diffusion properties in both GM and WM; even on a sub-millimeter scale in rat brain. In addition, these results show that the DTI findings have a positive correlation to the histopathological changes in HCP rats. Thus we establish that DTI parameters may be used as a surrogate marker to evaluate cerebral tissue integrity during the development of $\mathrm{HCP}$, further supporting its use in translational research studies in the clinical setting.

As summarized by Beaulieu et al. [6], anisotropic diffusion properties are strongly influenced by the microstructural components within the brain. The deviation of diffusion indices based on DTI from the normal range is believed to be an indication of the integrity, or the lack thereof, in various structural components, such as the myelin sheath or axonal membrane. Neuronal degeneration is often reported to be a reflection of decreased FA accompanied by an increased MD [36,37]. Conversely, increased FA accompanied by decreased radial diffusivity and increased axial diffusivity is often regarded as an indication of compression of tissue, as seen in spaceoccupying lesions, such as non-invasive tumors [38]. In clinical DTI studies of pediatric hydrocephalus, different patterns of change in DTI indices have been described in specific WM regions [18-21]. For example, infants with HCP have been reported to demonstrate abnormally low FA in the corpus callosum and abnormally high FA in the internal capsule [20]. Similarly, older patients ( $>7 \mathrm{yrs})$ also showed analogous patterns of diffusion [18]. We speculate that multiple injury mechanisms may coexist in the HCP patient. The clinical manifestation of injury in HCP may depend on various factors such as the location of the structure under study, and the intrinsic tissue resistance, as well as the timing and duration of $\mathrm{HCP}$ during critical time points of CNS development. Among the four WM regions of interest examined in the present study (Tables 2 and 3), the EC in most HCP rats was unidentifiable using DTI maps. This can be considered an indication of severe tissue damage. Even though anatomically identifiable during the preparation for immunohistochemical staining, partial volume effect prevented us from making meaningful measurements in this region in any of the HCP rats. In the CC and the IC, significant decreases in FA and increases in MD values were found in rats with HCP. The FX showed similar DTI trends even though the difference did not reach statistical significance. These group differences based on data averaged over time (between P7 and P12) can be found as early as P9 when the comparison was made for individual post natal days. This may serve as preliminary evidence of the early impact of HCP as reflected from the DTI measurement. As described earlier, a decrease in FA usually indicates degenerative damage in the myelin or axonal membrane [6], a change that is regarded as less reversible than a mere physical compression which often presents as an increase in FA $[38,39]$. Combining the early timing and the potential nature of the changes, our results support early management in the treatment of HCP. In the clinical setting it would translate to having a preoperative MRI demonstrating a significant reduction in FA values from our standard ROIs [20,21].

DTI is seldom used to quantify the anisotropy in GM structures. However, relatively high anisotropy has been reported previously in the neonatal brain of both experimental models and clinical studies [39-41]. In the normal individual, the elevated FA is followed by a rapid decrease until a plateau is reached in adulthood. This change in anisotropy is attributed to the developmental change in neuronal proliferation/migration, apoptosis and axonal pruning, and synaptogenesis occurring during the postnatal period [39]. In the present study, DTI was used to determine the anisotropic diffusion properties in three GM structures: the CX, CPu, and HC. When comparing our data to the FA values at a corresponding age reported by Bockhorst et al [39], our FA measurements in normal rats were similar in the CX but higher than previously reported in the $\mathrm{HC}$ and $\mathrm{CPu}$. In the present study, HCP rats had a significantly reduced FA (from 0.25 to 0.17 ) in the $C X$, indicating that the impact of HCP not only affects the adjacent periventricular WM and the subcortical WM, but also the GM structures that are further peripherally located within the cranial vault.

It should be stressed that the DTI measurement is an objective but indirect reflection of the underlying microstructural integrity or pathological condition under study. As a non-invasive imaging biomarker, DTI results 
need to be examined and correlated to the histopathological data which is regarded as the gold standard for studying the injury mechanisms at the cellular level. In this study, we examined GFAP immunoreactivity to characterize the temporal-spatial changes of reactive gliosis following HCP. Our results from GFAP stains showed that a strong glial reaction was seen in the HCP rats in the IC, EC, and FX at both P11 and P22/P23. Astrocytosis was also present at P11 in the CC. The correlation analysis of DTI with our GFAP ranking showed a moderate to strong correlation in the CC at P11. Likewise, the microglial reaction (Iba-1) also demonstrated a moderate to strong correlation with the DTI measurements in the CC at P11. LFB staining was very weak at P11 in control animals; this result is expected given that these observations occurred only three days after the initiation of myelin maturation (P7) $[39,42,43]$. Consequently, the paucity of LFB staining in hydrocephalic brains at P11 was difficult to interpret. At P22/P23, even though the reduction of myelination was, as expected, statistically significant in IC in HCP rats (Table 4), only a moderate correlation (without statistical significance) was found between the LFB staining in IC at P22/P23 and the DTI measurement at P7-P9 (Table 5).

In the present study, astrocytes and microglial cells demonstrated an early and continuous response to CNS injury in untreated HCP. The correlations between DTI and those cellular changes reveal initial evidence for the potential of DTI to serve as an imaging biomarker in studying the progression of HCP.

The results presented in this study add new important value to the field of advanced imaging in experimental HCP. However, caution should be exercised in the interpretation of the results due to the overall small sample size. Our data do not allow for a more comprehensive analysis to evaluate the causal effect or the predicting power of DTI. Moreover, it is possible that partial volume effects may have artificially increased the differences in diffusivity measurements between the groups. Within a $1.5 \mathrm{~mm}$ thick slice as used in the study, the structures examined may have changed diffusion direction. The impact of this potential confounding factor will be reduced by increasing the imaging resolution using $3 \mathrm{D}$ fast DTI imaging sequence, e.g., EPI or RARE based DTI sequence. With regard to the animal model, the current study will benefit if the progression of ventricular enlargement can be controlled and managed both in time and in the degree of severity. We have begun experiments to address these shortcomings. In addition, monitoring the longitudinal changes between the pre- and post-shunting conditions will further increase our understanding of the underlying injury mechanisms as well as the course for recovery.

\section{Conclusion}

The current study demonstrates that DTI is a sensitive tool in the investigation of brain structural integrity of the neonatal rat with hydrocephalus. Significant changes of diffusion properties (increased diffusivity and decreased anisotropy) occurred in the HCP rats in both GM and WM. DTI alterations significantly correlate with changes in astrocytosis, microgliosis, and myelination. These findings indicate that DTI is a useful imaging biomarker for non-invasive investigation of tissue injury and recovery in hydrocephalus.

\section{List of abbreviations}

CC: corpus callosum; CPu: caudate-putamen complex; CX: cortex; DTI: diffusion tensor imaging; EC:external capsule; FA: fractional anisotropy; FX: fornix; GFAP: glial fibrillary acidic protein; GM: grey matter; HC: hippocampus; HCP: hydrocephalus; Iba-1: ionized calcium binding adaptor molecule; IC: internal capsule; LFB: luxol fast blue; MD: mean diffusivity; WM: white matter.

\section{Acknowledgements}

This study was supported in part by the Robert L. McLaurin, MD, Faculty Development Scholarship in Neurosurgery at Cincinnati Children's Hospital Medical Center and the Primary Children Medical Center, University of Utah.

\section{Author details}

'Department of Radiology, Pediatric Neuroimaging Research Consortium, Cincinnati Children's Hospital Medical Center, University of Cincinnati College of Medicine, MLC 5033, 3333 Burnet Ave., Cincinnati, OH 45229, USA. ${ }^{2}$ Department of Neurosurgery, Division of Pediatric Neurosurgery, Primary Children's Medical Center and the University of Utah, N. Medical Drive East, Salt Lake City, UT 84132, USA. ${ }^{3}$ Department of Radiology, Imaging Research Center, Cincinnati Children's Hospital Medical Center and University of Cincinnati College of Medicine, MLC 5033, 3333 Burnet Ave., Cincinnati, OH 45229, USA. "Division of Pediatric Neurosurgery, University of Cincinnati, Cincinnati Children's Hospital Medical Center MLC 2016, 3333 Burnet Avenue, Cincinnati, OH 45229, USA. ${ }^{5}$ Department of Biostatistics \&

Epidemiology, Cincinnati Children's Hospital Medical Center, MLC 5041, 3333 Burnet Ave., Cincinnati, OH 45229, USA.

\section{Authors' contributions}

WY: conception and design, data processing and analysis, interpretation of data, manuscript drafting, revision, and finalizing; KED: data acquisition, data processing and analysis, manuscript revision; JPM: conception and design, data analysis, interpretation of data, manuscript drafting, revision, and finalizing; SKH: conception and design, interpretation of data, manuscript drafting and revision; DML: conception and design, data acquisition, manuscript drafting and revision; AC: data acquisition, manuscript drafting and revision; MM: data acquisition, data analysis, manuscript revision; AS: data acquisition, data processing, manuscript revision; DH: data acquisition, data interpretation, manuscript drafting and revision; MA: data analysis, manuscript revision; FTM: conception and design, data analysis, interpretation of data, manuscript drafting, revision, and finalizing. All authors have read and approved the final version of the manuscript.

\section{Competing interests}

The authors declare that they have no competing interests.

Received: 28 June 2010 Accepted: 5 November 2010 Published: 5 November 2010

\section{References}

1. Hirsch JF: Surgery of hydrocephalus: past, present and future. Acta Neurochir (Wien) 1992, 116:155-160.

2. Douek P, Turner R, Pekar J, Patronas N, Le Bihan D: MR color mapping of myelin fiber orientation. J Comput Assist Tomogr 1991, 15:923-929. 
3. Basser PJ, Mattiello J, LeBihan D: Estimation of the effective self-diffusion tensor from the NMR spin echo. J Magn Reson B 1994, 103:247-254.

4. Basser PJ, Mattiello J, LeBihan D: MR diffusion tensor spectroscopy and imaging. Biophys J 1994, 66:259-267.

5. Beaulieu C, Allen PS: Determinants of anisotropic water diffusion in nerves. Magn Reson Med 1994, 31:394-400.

6. Beaulieu C: The basis of anisotropic water diffusion in the nervous system - a technical review. NMR Biomed 2002, 15:435-455.

7. Horsfield MA, Jones DK: Applications of diffusion-weighted and diffusion tensor MRI to white matter diseases - a review. NMR Biomed 2002, 15:570-577.

8. Neil J, Miller J, Mukherjee P, Huppi PS: Diffusion tensor imaging of normal and injured developing human brain - a technical review. NMR Biomed 2002, 15:543-552.

9. Sotak $\mathrm{CH}$ : The role of diffusion tensor imaging in the evaluation of ischemic brain injury - a review. NMR Biomed 2002, 15:561-569.

10. Moseley ME, Cohen Y, Kucharczyk J, Mintorovitch J, Asgari HS, Wendland MF, Tsuruda J, Norman D: Diffusion-weighted MR imaging of anisotropic water diffusion in cat central nervous system. Radiology 1990, 176:439-445.

11. Henkelman RM, Stanisz GJ, Kim JK, Bronskill MJ: Anisotropy of NMR properties of tissues. Magn Reson Med 1994, 32:592-601.

12. Pierpaoli C, Jezzard P, Basser PJ, Barnett A, Di Chiro G: Diffusion tensor MR imaging of the human brain. Radiology 1996, 201:637-648.

13. Rosenberger $G$, Kubicki M, Nestor $P G$, Connor E, Bushell GB, Markant $D$, Niznikiewicz M, Westin CF, Kikinis R, A JS, et al: Age-related deficits in fronto-temporal connections in schizophrenia: a diffusion tensor imaging study. Schizophr Res 2008, 102:181-188.

14. Werring DJ, Clark CA, Barker GJ, Thompson AJ, Miller DH: Diffusion tensor imaging of lesions and normal-appearing white matter in multiple sclerosis. Neurology 1999, 52:1626-1632.

15. Werring DJ, Toosy AT, Clark CA, Parker GJ, Barker GJ, Miller DH, Thompson AJ: Diffusion tensor imaging can detect and quantify corticospinal tract degeneration after stroke. J Neurol Neurosurg Psychiatry 2000, 69:269-272.

16. Huisman TA, Schwamm LH, Schaefer PW, Koroshetz WJ, Shetty-Alva N, Ozsunar $Y$, Wu O, Sorensen AG: Diffusion tensor imaging as potential biomarker of white matter injury in diffuse axonal injury. AJNR Am J Neuroradiol 2004, 25:370-376.

17. Wilde EA, Chu Z, Bigler ED, Hunter JV, Fearing MA, Hanten G, Newsome MR, Scheibel RS, Li X, Levin HS: Diffusion tensor imaging in the corpus callosum in children after moderate to severe traumatic brain injury. I Neurotrauma 2006, 23:1412-1426.

18. Assaf $Y$, Ben-Sira L, Constantini $S$, Chang LC, Beni-Adani L: Diffusion tensor imaging in hydrocephalus: initial experience. AJNR Am J Neuroradiol 2006, 27:1717-1724.

19. Hasan KM, Eluvathingal TJ, Kramer LA, Ewing-Cobbs L, Dennis M, Fletcher JM: White matter microstructural abnormalities in children with spina bifida myelomeningocele and hydrocephalus: A diffusion tensor tractography study of the association pathways. J Mag Res Imag 2008, 27:700-709.

20. Yuan W, Mangano FT, Air EL, Holland SK, Jones BV, Altaye M, Bierbrauer K: Anisotropic diffusion properties in infants with hydrocephalus: a diffusion tensor imaging study. AJNR Am J Neuroradiol 2009, 30:1792-1798.

21. Air EL, Yuan W, Holland SK, Jones BV, Bierbrauer K, Altaye M, Mangano FT: Longitudinal comparison of pre- and postoperative diffusion tensor imaging parameters in young children with hydrocephalus. J Neurosurg Pediatr 2010, 5:385-391.

22. MCAllister JP, Maugans TA, Shah MV, Truex RC Jr: Neuronal effects of experimentally induced hydrocephalus in newborn rats. J Neurosurg 1985, 63:776-783.

23. DiFrancesco MW, Rasmussen JM, Yuan W, Pratt R, Dunn S, Dardzinski BJ, Holland SK: Comparison of SNR and CNR for in vivo mouse brain imaging at 3 and $7 \mathrm{~T}$ using well matched scanner configurations. Med Phys 2008, 35:3972-3978.

24. Freeman $\mathrm{GH}$, Halton $\mathrm{JH}$ : Note on an exact treatment of contingency, goodness of fit and other problems of significance. Biometrika 1951, 38:141-149.

25. Del Bigio MR, Wilson MJ, Enno T: Chronic hydrocephalus in rats and humans: white matter loss and behavior changes. Ann Neurol 2003, 53:337-346.

26. Khan $\mathrm{OH}$, Enno TL, Del Bigio MR: Brain damage in neonatal rats following kaolin induction of hydrocephalus. Exp Neurol 2006, 200:311-320.
27. Miller JM, McAllister JP: Reduction of astrogliosis and microgliosis by cerebrospinal fluid shunting in experimental hydrocephalus. Cerebrospinal Fluid Res 2007, 4:5.

28. Ito D, Imai Y, Ohsawa K, Nakajima K, Fukuuchi Y, Kohsaka S: Microgliaspecific localisation of a novel calcium binding protein, Iba1. Brain Res Mol Brain Res 1998, 57:1-9.

29. Deren KE, Forsyth J, Abdullah O, Hsu EW, Klinge PM, Silverberg GD, Johanson CE, MCAllister JP: Low levels of amyloid-beta and its transporters in neonatal rats with and without hydrocephalus. Cerebrospinal Fluid Res 2009, 6:4.

30. Deren KE, Packer M, Forsyth J, Milash B, Abdullah OM, Hsu EW, McAllister JP: Reactive astrocytosis, microgliosis and inflammation in rats with neonatal hydrocephalus. Exp Neurol 2010, 226:110-119.

31. Yuan W, Holland SK, Schmithorst VJ, Walz NC, Cecil KM, Jones BV, Karunanayaka P, Michaud L, Wade SL: Diffusion tensor MR imaging reveals persistent white matter alteration after traumatic brain injury experienced during early childhood. AJNR Am J Neuroradiol 2007, 28:1919-1925.

32. Yuan W, Holland SK, Jones BV, Crone K, Mangano FT: Characterization of abnormal diffusion properties of supratentorial brain tumors: a preliminary diffusion tensor imaging study. J Neurosurg Pediatr 2008, 1:263-269.

33. Jiang H, van Zijl PC, Kim J, Pearlson GD, Mori S: DtiStudio: resource program for diffusion tensor computation and fiber bundle tracking. Comput Methods Programs Biomed 2006, 81:106-116.

34. Box GEP, Cox DR: An analysis of transformations. J R Stat Soc, Ser B 1964, 26:211-252.

35. Benjamini $Y$, Hochberg Y: Controlling the false discovery rate: a practical and powerful approach to multiple testing. J Roy Stat Soc Ser B 1995, 57:1919-1925.

36. Wang J, Wai Y, Lin WY, Ng S, Wang CH, Hsieh R, Hsieh C, Chen RS, Lu CS: Microstructural changes in patients with progressive supranuclear palsy: a diffusion tensor imaging study. J Magn Reson Imaging 2010, 32:69-75.

37. Boska MD, Hasan KM, Kibuule D, Banerjee R, McIntyre E, Nelson JA, Hahn T, Gendelman HE, Mosley RL: Quantitative diffusion tensor imaging detects dopaminergic neuronal degeneration in a murine model of Parkinson's disease. Neurobiol Dis 2007, 26:590-596.

38. Schonberg T, Pianka P, Hendler T, Pasternak O, Assaf Y: Characterization of displaced white matter by brain tumors using combined DTI and fMRI. Neuroimage 2006, 30:1100-1111.

39. Bockhorst KH, Narayana PA, Liu R, Ahobila-Vijjula P, Ramu J, Kamel M, Wosik J, Bockhorst T, Hahn K, Hasan KM, Perez-Polo JR: Early postnatal development of rat brain: in vivo diffusion tensor imaging. J Neurosci Res 2008, 86:1520-1528.

40. McKinstry RC, Mathur A, Miller JH, Ozcan A, Snyder AZ, Schefft GL, Almli CR, Shiran SI, Conturo TE, Neil JJ: Radial organization of developing preterm human cerebral cortex revealed by non-invasive water diffusion anisotropy MRI. Cereb Cortex 2002, 12:1237-1243.

41. Maas LC, Mukherjee P, Carballido-Gamio J, Veeraraghavan S, Miller SP, Partridge SC, Henry RG, Barkovich AJ, Vigneron DB: Early laminar organization of the human cerebrum demonstrated with diffusion tensor imaging in extremely premature infants. Neuroimage 2004, 22:1134-1140.

42. Hamano K, Iwasaki N, Takeya T, Takita H: A quantitative analysis of rat central nervous system myelination using the immunohistochemical method for MBP. Brain Res Dev Brain Res 1996, 93:18-22.

43. Hamano K, Takeya T, Iwasaki N, Nakayama J, Ohto T, Okada Y: A quantitative study of the progress of myelination in the rat central nervous system, using the immunohistochemical method for proteolipid protein. Brain Res Dev Brain Res 1998, 108:287-293.

doi:10.1186/1743-8454-7-19

Cite this article as: Yuan et al:: Diffusion tensor imaging correlates with cytopathology in a rat model of neonatal hydrocephalus. Cerebrospinal Fluid Research 2010 7:19. 